\title{
AMCoR
}

Asahikawa Medical College Repository http://amcor.asahikawa-med.ac.jp/

The Journal of urology (2003) 169(2):655-658.

Diameter of the external urethral sphincter as a predictor of detrusorsphincter incoordination in children:Comparative study of voiding cystourethrography

HIDEHIRO, KAKIZAKI ; KIMIHIKO, MORIYA ; KANAME, AMEDA ; TAKASHI, SHIBATA ; HIROSHI, TANAKA ; TOMOHIKO, KOYANAGI 


\section{DIAMETER OF THE EXTERNAL URETHRAL SPHINCTER AS A PREDICTOR OF DETRUSOR-SPHINCTER INCOORDINATION IN CHILDREN: COMPARATIVE STUDY OF VOIDING CYSTOURETHROGRAPHY}

HIDEHIRO KAKIZAKI, KIMIHIKO MORIYA, KANAME AMEDA, TAKASHI SHIBATA， HIROSHI TANAKA， AND TOMOHIKO KOYANAGI

From the Department of Urology, Hokkaido University Graduate School of Medicine,

$$
\text { Sapporo, Japan }
$$

Brief running head: Voiding cystourethrography in children

Key Words: voiding dysfunction, urethra, sphincter, children, urodynamics

Correspondence to: Hidehiro Kakizaki, M.D.

Department of Urology,

Hokkaido University Graduate School of Medicine

North-15 West-7 Kita-Ku, Sapporo 060-8638, Japan

Phone: +81-11-716-1161 ext.5949

Fax: +81-11-706-7853

E-mail: kaki@med.hokudai.ac.jp 


\section{ABSTRACT}

Purpose: Voiding cystourethrography (VCUG) is one of diagnostic procedures widely used to evaluate lower urinary tract abnormalities in children. For children with or without suspected voiding dysfunction, we measured the internal diameter of the external urethral sphincter (EUSD) on VCUG to evaluate its diagnostic accuracy as a predictor of detrusor-sphincter incoordination.

Materials and Methods: (Part 1) Ninety-six children (59 boys and 37 girls) with normal voiding function underwent VCUG. In each child, 1 to 6 VCUGs (mean 2.1) were performed as a part of urological evaluations. Thus, a total of 200 VCUGs were collected. Underlying urological disease was primary vesicoureteral reflux in 57, congenital hydronephrosis in 9, urinary tract infection in 6, ureteral anomalies in 11, and others in 13. For the determination of EUSD in a given VCUG, EUSD was measured in consecutive films and the widest EUSD was chosen. (Part 2) Forty-three children with suspected voiding dysfunction underwent VCUG and sphincter electromyography (EUS-EMG). The findings of EUS-EMG were comparatively analyzed with EUSD.

Results: (Part 1) Age-dependent increase in EUSD was seen in children with normal voiding function. EUSD (mm) was formulated as $0.166 \mathrm{x}$ age (years) +4.31 for boys $(\mathrm{p}=0.0001, \mathrm{r}=0.374)$, and $0.222 \mathrm{x}$ age (years $)+2.73$ for girls $(\mathrm{p}<0.0001, \mathrm{r}=0.595)$. Overall incidence of EUSD less than 3 mm was only 4\% (8/200). (Part 2) EUS-EMG documented detrusor-sphincter incoordination in 15 children (35\%). When we defined EUSD less than 3mm as a cut-off value for predicting detrusor-sphincter incoordination, the sensitivity, specificity, positive and negative predictive values were 93\%, 89\%, 82\% and $96 \%$, respectively.

Conclusions: EUSD has a satisfactory sensitivity and specificity as a predictor of 
detrusor-sphincter incoordination in children. Thus, simple measurement of EUSD on VCUG should be recommended in children with suspected voiding dysfunction before employing rather invasive urodynamic study. 


\section{INTRODUCTION}

If children having lower urinary tract symptoms with or without urinary tract infection visit urologists, and if these children do not have upper urinary tract anomalies, we need to differentiate structural and functional lower urinary tract abnormalities. Simple but careful observation of their voiding alone can be a good clue to determine voiding disorders in some cases. However, radiological and/or endoscopic assessments can be more informative, and if indicated, urodynamic study is performed. Most of all, voiding cystourethrography (VCUG), which is one of the most widely used procedures in the evaluation of lower urinary tract abnormalities, would be the best method to indirectly diagnose structural or functional abnormalities of the lower urinary tract. ${ }^{1-3}$ However, the possibility of quantitative evaluation of VCUG for the external urethral sphincter (EUS) function has not been previously mentioned.

Functional urethral obstruction by detrusor-sphincter incoordination is a major cause of voiding disorders in children. It can occur not only in children with overt neurological deficits (eg. myelomeningocele) but also in those neurologically normal children. ${ }^{4-7}$ Electromyography of the EUS (EUS-EMG) is one of the most reliable methods for diagnosing detrusor-sphincter incoordination. EUS-EMG by introduction of a fine needle electrode to the EUS is no doubt the most accurate way to detect detrusor-sphincter incoordination. $^{8,9}$ However, it is extremely hard for small children with normal sacral sensation to be patient throughout the EUS-EMG study. Thereby, some recommend the use of a patch electrode. Unfortunately, activity of the pelvic floor musculature evaluated by patch electrodes does not always reflect the EUS activity. ${ }^{10}$

To promote a diagnostic accuracy of VCUG for detrusor-sphincter incoordination, we carried out 2 studies. If the urethral sphincter fully relaxes during voiding, it would give an 
unobstructive radiological appearance of the sphincteric urethra on VCUG. Thus, the hypothesis was that normal EUS relaxation during voiding would result in a wider internal diameter of the EUS on VCUG than that of the children with sphincter incoordination. In the study of part 1, we measured the internal diameter of the EUS (EUSD) on VCUG in children without voiding dysfunction, and analyzed age-dependent distributions of EUSD. In part 2, children with suspected voiding dysfunction underwent VCUG as well as EUS-EMG, and the findings of EUS-EMG were comparatively analyzed with EUSD. Through these investigations, we proposed a diagnostic cut-off value of EUSD as a predictor of detrusor-sphincter incoordination in children. 


\section{MATERIALS AND METHODS}

(Part 1) Ninety-six neurologically normal children without voiding dysfunction underwent VCUG and endoscopy as a part of urological evaluations for various urological disorders that included primary vesicoureteral reflux (VUR) in 57, congenital hydronephrosis in 9, urinary tract infection in 6, ureteral anomalies in 11, and others in 13. In children who finished toilet training, a careful history taking on voiding behavior was performed. Those children with suspected dysfunctional voiding, significant bladder deformity or dilated posterior urethra on VCUG, or organic urethral abnormalities on endoscopy were not included in the study. In each child, 1 to 6 VCUGs (mean 2.1) were performed on different occasions (at least 1 year apart), and thus a total of 200 VCUGs were collected. Ages at the time of VCUG were 1 week to 11 years (mean 4.9 years). VCUG was performed without any anesthetic or sedative agents. Spot films were taken of the bladder before and of the bladder and urethra during voiding. During voiding, the patients were placed in an oblique position for better visualization of the urethra. For measurement of EUSD, a cm ruler was shown on all films as a template guide. To determine EUSD in a given VCUG, EUSD was measured in every consecutive film, and the widest EUSD was chosen and expressed by 0.5 mm (Fig.1). Correlation between age and EUSD was analyzed with Spearman's rank correlation.

(Part 2) At our clinic, children with voiding problems are initially screened by a careful history taking, urine analysis, and ultrasonographic evaluation of the upper urinary tract and postvoid residual urine. Following these assessments, VCUG is carried out for children with suspected lower urinary tract abnormalities and/or urinary tract infection. Uroflowmetry and filling cystometry are also performed in older children if indicated. 
When these examinations show some abnormalities, appropriate urinary management with or without medication is initiated. If there are persistent voiding problems, upper urinary tract deterioration or recurrent urinary tract infection, more extensive evaluations are discussed with parents and planned. According to this clinical strategy, the combined assessment of endoscopy and EUS-EMG was indicated for 43 children who were suspected to have outlet obstruction but ambiguous in origin. There were 25 boys and 18 girls, age ranging 2 months to 13 years (mean 4.5 years). Underlying disorders were lumbosacral lipoma in 11, tethered spinal cord in 6 , myelomeningocele in 5 , imperforate anus in 4 , tight filum terminale, mental retardation and central nervous system anomaly in 1 each, while remaining 23 children were without any overt neurological deficits. Six children had more than 1 disorder. Before examinations, informed consents were obtained from all parents or guardians.

Endoscopic examinations and EUS-EMG were done with the patients under general anesthesia, which consisted of nitrous oxide and sevoflurane without premedication with atropine. Detailed procedures have been described in a previous report. ${ }^{11}$ Following endoscopic evaluation of the bladder and urethra to rule out structural abnormalities, an 8 Fr. double lumen catheter and a needle electrode were introduced to the bladder and EUS, respectively, and intravesical pressure and EUS-EMG activity were monitored simultaneously during bladder filling and emptying, while the level of anesthesia was tapered off. These urodynamic studies with the patients under general anesthesia correlate well with awake studies. ${ }^{12}$ Detrusor-sphincter incoordination was defined as failure of EUS activity to decrease during bladder contraction or sustained increase in intravesical pressure. ${ }^{4}$ The findings of EUS-EMG were analyzed by the investigators who were blinded to EUSD. Then the results of EUS-EMG were compared with EUSD. 


\section{RESULTS}

(Part 1) Age-dependent increase in EUSD was seen in both boys (Fig. 2) and girls (Fig. 3). Regression line indicated that EUSD $(\mathrm{mm})$ was $[0.166 \mathrm{x}$ age (years) +4.31$]$ for boys $(p=0.0001, r=0.374)$, and [0.222 $x$ age (years $)+2.73]$ for girls $(p<0.0001, r=0.595)$. As revealed in Fig. 2 and 3, a great majority of children had EUSD of $3 \mathrm{~mm}$ or more. Incidence of EUSD less than $3 \mathrm{~mm}$ was only $0.8 \%$ (1/124) in boys, 9.2\% (7/76) in girls, and $4 \%(8 / 200)$ in overall.

(Part 2) Detrusor-sphincter incoordination was documented in 15 children (35\%, 8 boys and 7 girls) while absent in other 28 (65\%, 17 boys and 11 girls). Underlying disorders in children with sphincter incoordination were lumbosacral lipoma in 4, myelomeningocele in 4 , imperforate anus in 2, mental retardation and nervous system anomaly in 1 each, and unknown in 3. EUSD in children with sphincter incoordination $(2.0 \pm 0.9 \mathrm{~mm})$ was significantly smaller than in those without sphincter incoordination $(4.6 \pm 1.8 \mathrm{~mm})$ (unpaired t test, $\mathrm{p}<0.00001$ ). Age and EUSD in each child was plotted in Fig. 4. Based on the results of part 1 study, if we simply defined EUSD less than $3 \mathrm{~mm}$ as a cut-off value for predicting detrusor-sphincter incoordination, 14 of the 15 children with detrusor-sphincter incoordination had EUSD less than $3 \mathrm{~mm}$, while 25 of the 28 children without detrusor-sphincter incoordination had EUSD $3 \mathrm{~mm}$ or greater. Thus, the sensitivity, specificity, positive and negative predictive values of EUSD were 93\% (14/15), 89\% (25/28), 82\% (14/17), and 96\% (25/26), respectively. 


\section{DISCUSSION}

We evaluated the possibility of detecting detrusor-sphincter incoordination with VCUG in children with suspected voiding dysfunction. When we simply defined EUSD less than 3mm as a cut-off value, EUSD had the satisfactory sensitivity and specificity for detecting detrusor-sphincter incoordination in children. Thus, a simple measurement of EUSD is recommended in children with suspected voiding dysfunction when VCUG is performed as a part of initial diagnostic assessments.

Differential diagnosis of structural and functional lower urinary tract abnormalities in children is not always easy only by a careful history taking and a screening ultrasound. VCUG is routinely performed in children with urinary tract infection, particularly to diagnose vesicoureteral reflux and exclude urinary tract anomalies. VCUG is also often indicated in children with voiding problems to rule out organic urethral obstruction. Thus, VCUG has been used in routine urology practice settings to visualize the lower urinary tract for evaluating the presence or absence of urinary tract abnormalities. VCUG also has a different role in estimating bladder and urethral function during urine storage and voiding. A recent study demonstrated the possibility of identifying bladder instability with VCUG in infants. $^{13}$ Unstable detrusor contractions could be identified in infants by evaluation of 3 radiological signs on the filling phase of VCUG, including bladder wall irregularity, elongation of bladder shape and filling of the posterior urethra. The authors propose that radiological diagnosis of detrusor instability during filling can be an indication of the need for further assessment with cystometry. However, the possibility of detecting detrusor-sphincter incoordination on VCUG has not been fully explored.

Estimation of voiding function on VCUG is made by assessing bladder shape, funneling configuration of the trigone and bladder neck, appearance of the urethra, urine stream and 
postvoid residual urine. The most conventional radiological signs for functional urethral obstruction have been the presence of bladder trabeculation, posterior urethral dilatation and postvoid residual urine. They might be enough to help the precise judgment. However, these assessments are usually qualitative rather than quantitative. If the urethral sphincter fully relaxes during voiding, it would give an unobstructive, non-dilated radiological appearance of the posterior urethra and adequate width of the sphincteric urethra on VCUG. When we applied a unique parameter of EUSD to the evaluation of urethral sphincter function, the diagnostic value of VCUG obviously increased. Children with detrusor-sphincter incoordination showed narrower EUSD than those without abnormal sphincter function ( $2.0 \pm 0.9$ vs $4.6 \pm 1.8 \mathrm{~mm})$. Because $96 \%$ of children with normal voiding function had EUSD $3 \mathrm{~mm}$ or greater, we simply defined EUSD less than $3 \mathrm{~mm}$ as a cut-off value for detecting detrusor-sphincter incoordination. This cut-off value of EUSD had the satisfactory sensitivity (93\%), specificity (89\%), positive (82\%) and negative predictive values (96\%) among children with suspected voiding dysfunction. Taking into account the age-dependent increase in EUSD in children with normal voiding function, the diagnostic cut-off value should ideally be an age-dependent variable. However, a simpler cut-off value is more preferable in routine urology practice settings if only it has a reasonable diagnostic accuracy. Thereby, we propose EUSD less than $3 \mathrm{~mm}$ as a cut-off value for predicting detrusor-sphincter incoordination in children.

The most ideal study for children with suspected voiding dysfunction is videourodynamics that enables radiological as well as urodynamic functional evaluations of the lower urinary tract at the same time. However, videourodynamics is only performed at specialized centers and is reserved for selected or referred cases to explain the pathophysiology of voiding dysfunction. Thus, it is important to evaluate the reliability of VCUG for detecting detrusor-sphincter incoordination in children. We believe EUSD less 
than $3 \mathrm{~mm}$ should be an indication of the need for further assessment with detailed urodynamics.

It is well known that children with primary VUR often have detrusor instability. ${ }^{14,15}$ Even when children with severe dysfunctional voiding and urge incontinence were excluded, still about $20 \%$ of children with primary VUR were thought to have bladder/sphincter dysfunction. ${ }^{16}$ Urodynamic investigations in infants with VUR revealed an interesting aspect of pathophysiology of VUR in this very young age group of children. ${ }^{17-19}$ Bladder/sphincter dysfunction or immaturity as the form of detrusor instability, inadequate detrusor, detrusor-sphincter incoordination or obstructive high pressure voiding is strongly implicated as causative factors of VUR in infants. ${ }^{18}$ Furthermore, voiding dysfunction is suggested to be an integral part of the ectopic ureterocele complex. ${ }^{20}$ Thus, in urology practice there are numerous situations where the functional evaluation of the bladder and sphincter is desired for children with urinary tract abnormalities. Videourodynamics would be the most ideal method for functional evaluation of the bladder and urethra. However, that is only performed in specialized centers with full equipments of examinations. Instead, as shown in the current study, quantitative evaluation of VCUG by using EUSD may be a reasonable alternative to speculate the urethral sphincter function during voiding. EUSD less than $3 \mathrm{~mm}$ should be an indication of the need for detailed urodynamics. When detailed urodynamics is not available, simple cystometry and EUSD measurement on VCUG, with or without uroflowmetry, may be a minimal requirement for children in whom functional evaluation of the bladder and sphincter is indicated because of suspected voiding dysfunction or the presence of urinary tract abnormalities. 


\section{CONCLUSIONS}

We evaluated the possibility of detecting detrusor-sphincter incoordination with VCUG in children with suspected voiding dysfunction. When we simply defined EUSD less than 3mm as a cut-off value, EUSD had the satisfactory sensitivity and specificity for detecting detrusor-sphincter incoordination in children. Although a great care should be taken for the children to avoid overexposure to radiation, the informative values of VCUG can outweigh such adverse problems. We conclude that a simple measurement of EUSD is recommended in children with suspected voiding dysfunction when VCUG is performed as a part of initial diagnostic assessments. 


\section{REFERENCES}

1. Papanicolaou, N.: Urinary tract imaging and intervention: Basic principles. In Campbell's urology, $7^{\text {th }}$ edition. Edited by Walsh, P.C., Retik, A.B., Vaughan, Jr., E.D. and Wein, A.J., Philadelphia, W.B. Saunders Co., volume 1, chap. 6, 170-260, 1998.

2. Zawin, J.K. and Lebowitz, R.L.: Neurogenic dysfunction of the bladder in infants and children: Recent advances and the role of radiology. Radiology, 182: 297, 1992.

3. Koyanagi, T.: The role of voiding cysto-urethrography (VCU) in urological practice. Jpn. J. Urol., 65: 29, 1974.

4. Bauer, S.B., Hallett, M., Khoshbin, S. et al: Predictive value of urodynamic evaluation in newborns with myelodysplasia. J.A.M.A., 252: 650, 1984.

5. Hinman, Jr., F.: Nonneurogenic neurogenic bladder (the Hinman syndrome)- 15 years later. J. Urol., 136: 769, 1986.

6. Bauer, S.B., Dyro, F.M., Krarup, C. et al: The unrecognized neuropathic bladder of infancy. J. Urol., 142: 589, 1989.

7. Jayanthi, V.R., Khoury, A.E., McLorie, G.A. et al: The nonneurogenic neurogenic bladder of early infancy. J. Urol., 158: 1281, 1997.

8. Blaivas, J.G.: Sphincter electromyography. Neurourol. Urodyn., 2: 269, 1983.

9. Siroky, M.B.: Electromyography of the perineal striated muscles. In Clinical Neuro-Urology, $2^{\text {nd }}$ edition. Edited by Krane, R.J. and Siroky, M.B., Boston, Little, Brown and Co., chap. 12, 245-254, 1991.

10. Koyanagi, T., Arikado, K., Takamatsu, T. et al: Experience with electromyography of the external urethral sphincter in spinal cord injury patients. J. Urol., 127: 272, 1982.

11. Ameda, K., Kakizaki, H., Yamashita, T. et al: Feasibility of urodynamic study (combined cystometry and electromyography of the external urethral sphincter) under 
general anesthesia in children. Int. J. Urol., 4: 32, 1997.

12. Kakizaki, H., Nonomura, K., Asano, Y. et al: Preexisting neurogenic voiding dysfunction in children with imperforate anus: Problems in management. J. Urol., 151: 1041, 1994.

13. Bachelard, M., Verkauskas, G., Bertilsson, M. et al: Recognition of bladder instability on voiding cystourethrography in infants with urinary tract infection. J. Urol., 166: 1899, 2001.

14. Koyanagi, T., Ishikawa, T. and Tsuji, I.: Vesico-ureteral reflux and uninhibited neurogenic bladder. Int. Urol. Nephrol., 9: 217, 1977.

15. Koff, S.A. and Murtagh, D.S.: The uninhibited bladder in children: Effect of treatment on recurrence of urinary tract infection and on vesicoureteral reflux resolution. J. Urol., 130: 1138, 1983.

16. Van Gool, J.D., Hjalmas, K., Tamminen-Mobius, T. et al: Historical clues to the complex of dysfunctional voiding, urinary tract infection and vesicoureteral reflux. J. Urol., 148: 1699, 1992.

17. Sillen, U., Bachelard, M., Hermanson, G. et al: Gross bilateral reflux in infants: Gradual decrease of initial detrusor hypercontractility. J. Urol., 155: 668, 1996.

18. Yeung, C.K., Godley, M.L., Dhillon, H.K. et al: Urodynamic patterns in infants with normal lower urinary tracts or primary vesico-ureteric reflux. Brit. J. Urol., 81: 498, 1998.

19. Chandra, M. and Maddix, H.: Urodynamic dysfunction in infants with vesicoureteral reflux. J. Pediatr., 136: 754, 2000.

20. Abrahamsson, K., Hansson, E., Sillen, U. et al: Bladder dysfunction: An integral part of the ectopic ureterocele complex. J. Urol., 160: 1468, 1998. 


\section{FIGURE LEGENDS}

Fig. 1 Schemes for measuring internal diameter of external urethral sphincter (EUSD) on voiding cystourethrography

Fig. 2 Relationship of age and EUSD in boys. Solid line indicates regression line. Dotted line indicates $3 \mathrm{~mm}$ of EUSD.

Fig. 3 Relationship of age and EUSD in girls. Solid line indicates regression line. Dotted line indicates $3 \mathrm{~mm}$ of EUSD.

Fig. 4 Relationship of age and EUSD in 43 children with suspected voiding dysfunction. Solid square and open circle indicate children with and without detrusor-sphincter incoordination, respectively. 
Figure 1

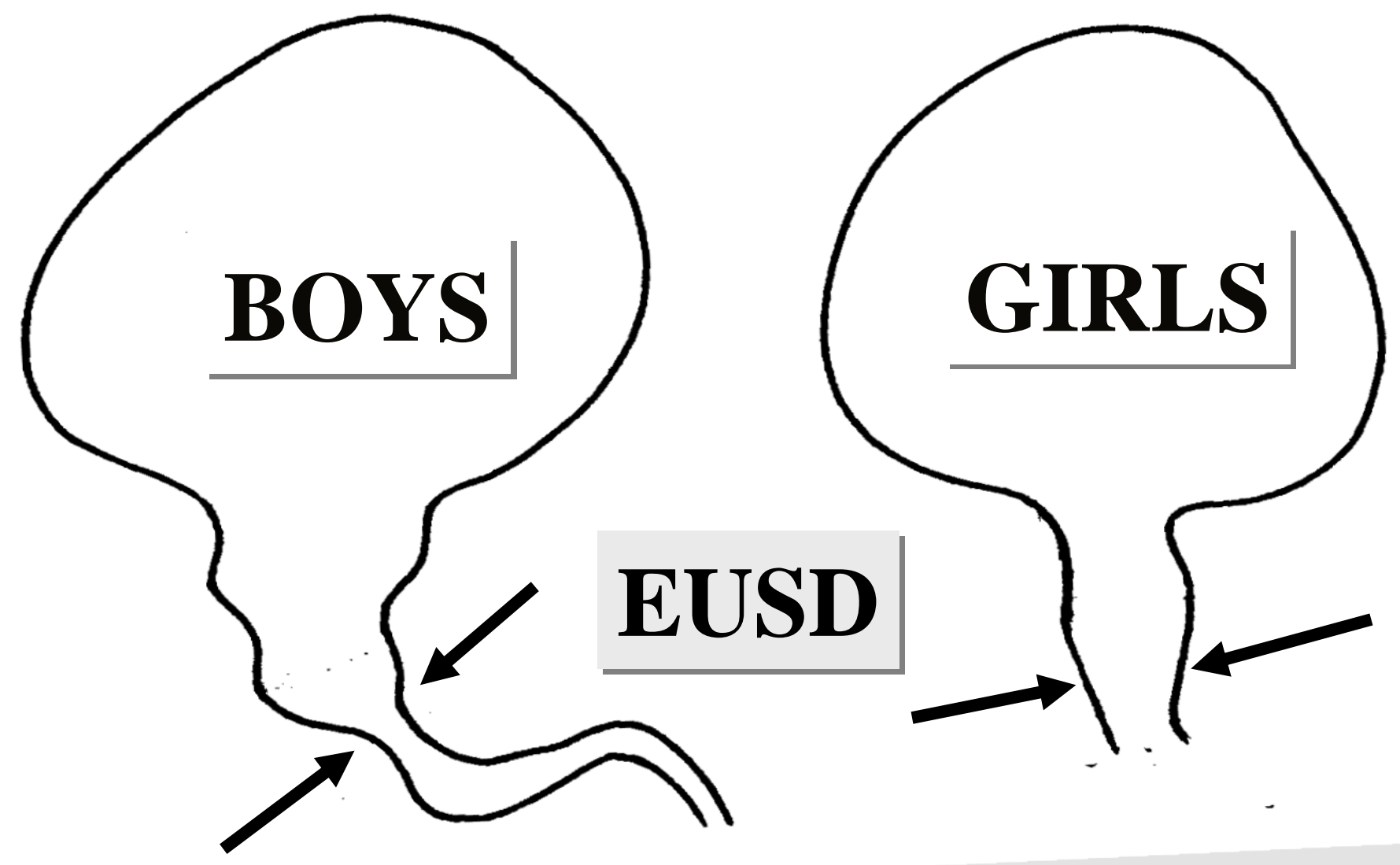


Figure 2

EUSD

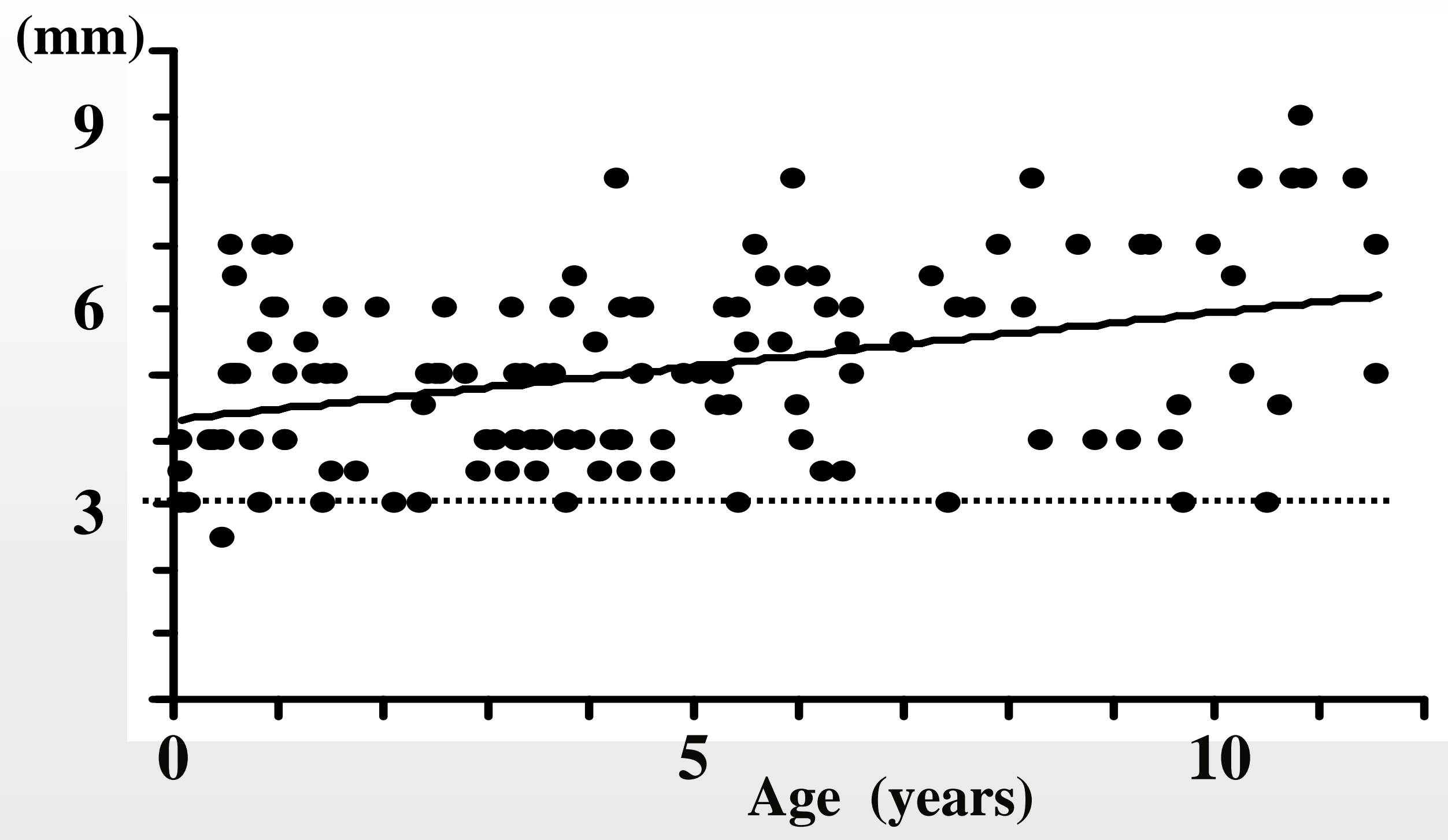


Figure 3

\section{EUSD}

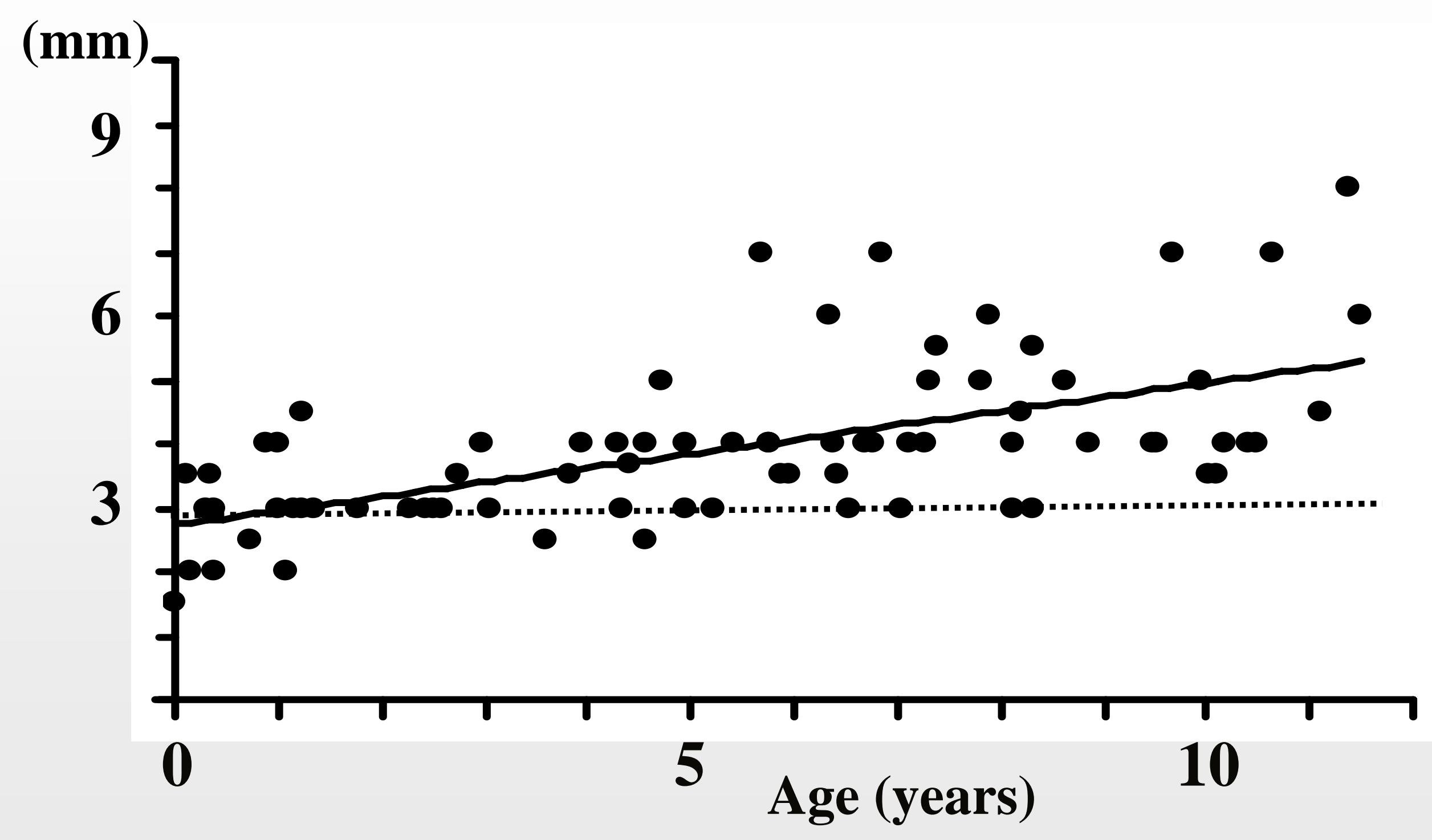


Figure 4

\section{EUSD}

(mm)

95

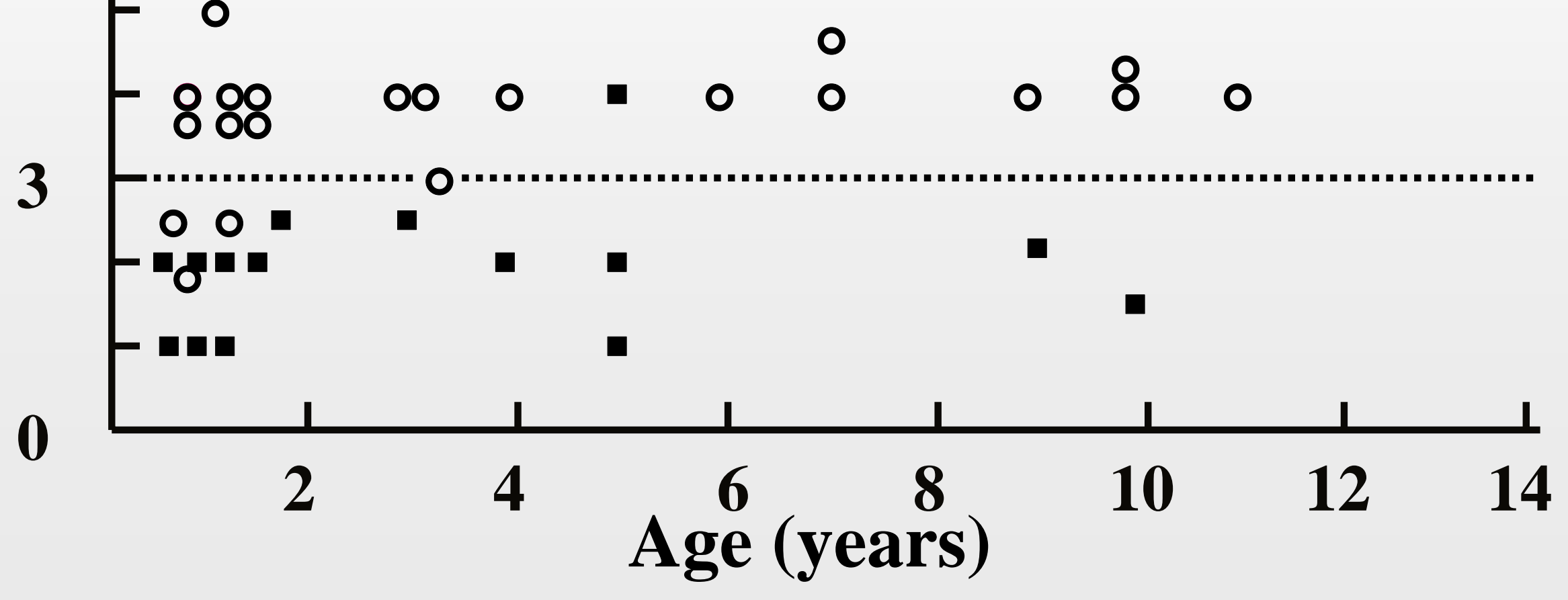

